\title{
Extreme Points of the Unit Ball in the Dual Space of Some Real Subspaces of Banach Spaces of Lipschitz Functions
}

\author{
Davood Alimohammadi and Hadis Pazandeh \\ Department of Mathematics, Faculty of Science, Arak University, Arak 38156-8-8349, Iran \\ Correspondence should be addressed to Davood Alimohammadi, d-alimohammadi@araku.ac.ir
}

Received 20 October 2011; Accepted 16 November 2011

Academic Editor: C. Zhu

Copyright (C) 2012 D. Alimohammadi and H. Pazandeh. This is an open access article distributed under the Creative Commons Attribution License, which permits unrestricted use, distribution, and reproduction in any medium, provided the original work is properly cited.

Let $X$ be a compact Hausdorff space, $\tau$ be a continuous involution on $X$ and $C(X, \tau)$ denote the uniformly closed real subalgebra of $C(X)$ consisting of all $f \in C(X)$ for which $f \circ \tau=\bar{f}$. Let $(X, d)$ be a compact metric space and let $\operatorname{Lip}\left(X, d^{\alpha}\right)$ denote the complex Banach space of complexvalued Lipschitz functions of order $\alpha$ on $(X, d)$ under the norm $\|f\|_{X, p_{\alpha}}=\max \left\{\|f\|_{X}, p_{\alpha}(f)\right\}$, where $\alpha \in(0,1]$. For $\alpha \in(0,1)$, the closed subalgebra of $\operatorname{Lip}(X, \alpha)$ consisting of all $f \in \operatorname{Lip}\left(X, d^{\alpha}\right)$ for which $|f(x)-f(y)| / d^{\alpha}(x, y) \rightarrow 0$ as $d(x, y) \rightarrow 0$, denotes by $\operatorname{lip}\left(X, d^{\alpha}\right)$. Let $\tau$ be a Lipschitz involution on $(X, d)$ and define $\operatorname{Lip}\left(X, \tau, d^{\alpha}\right)=\operatorname{Lip}\left(X, d^{\alpha}\right) \cap C(X, \tau)$ for $\alpha \in(0,1]$ and $\operatorname{lip}\left(X, \tau, d^{\alpha}\right)=$ $\operatorname{lip}\left(X, d^{\alpha}\right) \cap C(X, \tau)$ for $\alpha \in(0,1)$. In this paper, we give a characterization of extreme points of $B_{A^{*}}$, where $A$ is a real linear subspace of $\operatorname{Lip}\left(X, d^{\alpha}\right)$ or $\operatorname{lip}\left(X, d^{\alpha}\right)$ which contains 1 , in particular, $\operatorname{Lip}\left(X, \tau, d^{\alpha}\right)$ or $\operatorname{lip}\left(X, \tau, d^{\alpha}\right)$.

\section{Introduction and Preliminaries}

We let $\mathbb{R}, \mathbb{C}$, and $\mathbb{T}=\{z \in \mathbb{C}:|z|=1\}$ denote the field of real numbers, complex numbers, and the unit circle, respectively. The symbol $\mathbb{K}$ denotes a field that can be either $\mathbb{R}$ or $\mathbb{C}$. The elements of $\mathbb{K}$ are called scalars.

Let $\mathfrak{X}$ be a normed space over $\mathbb{K}$. We denote by $\mathfrak{X}^{*}$ and $B_{\mathfrak{X}}$ the dual space $\mathfrak{X}$ and the closed unit ball of $\mathfrak{X}$, respectively. If $S$ is a subset $\mathfrak{X}$, let $\operatorname{Ext}(S)$ denote the set of all extreme points of $S$. Let $A$ be a subspace of $\mathfrak{X}$ and $\varphi \in A^{*}$. A Hahn-Banach extension of $\varphi$ to $\mathfrak{X}$ is a continuous linear functional $\psi \in \mathfrak{X}^{*}$ such that $\left.\psi\right|_{A}=\varphi$ and $\|\psi\|=\|\varphi\|$. The set of all HahnBanach extensions of $\varphi$ to $\mathfrak{X}$ will be denoted by $H_{\varphi}$.

It is easy to see that if $\mathfrak{X}$ and $\mathcal{y}$ are normed spaces over $\mathbb{K}$ and $T: \mathfrak{X} \rightarrow \mathcal{y}$ is a linear isometry from $\mathfrak{X}$ onto $\mathcal{Y}$, then $T$ is a bijection mapping between $\operatorname{Ext}\left(B_{\mathfrak{X}}\right)$ and $\operatorname{Ext}\left(B_{\mathfrak{y}}\right)$. 
For a complex normed space $\mathfrak{X}$, we assume that $\mathfrak{X}_{r}$ denotes $\mathfrak{X}$, regarded as a real normed space by restricting the scalar multiplication to real numbers.

Kulkarni and Limaye gave some conditions for $\varphi \in B_{A^{*}}$ to be an extreme point of $B_{A^{*}}$ in terms of the Hahn-Banach extension of $\varphi$ to $\mathfrak{X}$ and the extreme points of $B_{\mathfrak{X}^{*}}$ as the following.

Theorem 1.1 (see [1, Theorem 2]). Let $\mathfrak{X}$ be a normed space over $\mathbb{K}, A$ be a nonzero linear subspace of $\mathfrak{X}$ and $\varphi \in B_{A^{*}}$.

(a) Let $\varphi \in \operatorname{Ext}\left(B_{A^{*}}\right)$. Then,

$$
H_{\varphi} \cap \operatorname{Ext}\left(B_{\mathfrak{X}^{*}}\right)=\operatorname{Ext}\left(H_{\varphi}\right) \neq \emptyset
$$

In particular, $\varphi$ has an extension to some $\psi \in \operatorname{Ext}\left(B_{\mathfrak{X}^{*}}\right)$. Further, if such an extension is unique, then $\varphi$ has a unique Hahn-Banach extension to $\mathfrak{X}$.

(b) Assume that whenever $\psi \in \operatorname{Ext}\left(B_{\mathfrak{X}^{*}}\right)$ and $\psi(f)=1$ for all $f \in A$ with $\varphi(f)=1=\|f\|$, one has $\left.\psi\right|_{A}=\varphi$, then $\varphi \in \operatorname{Ext}\left(B_{A^{*}}\right)$.

(c) If $\varphi$ has a unique Hahn-Banach extension $\psi$ to $\mathfrak{X}$ and if $\psi \in \operatorname{Ext}\left(B_{\mathfrak{X}^{*}}\right)$, then $\varphi \in \operatorname{Ext}\left(B_{A^{*}}\right)$.

Let $X$ be a compact Hausdorff space. We denote by $C(X)$ the complex Banach algebra of all continuous complex-valued functions on $X$ under the uniform norm $\|f\|_{X}=$ $\sup \{|f(x)|: x \in X\}$. For $x \in X$, consider the evaluation functional $e_{x}$ given by $e_{x}(f)=$ $f(x), f \in C(X)$. Clearly, $\lambda e_{x} \in B_{C(X)^{*}}$ for all $(x, \lambda) \in X \times \mathbb{T}$. It is well known [2, page 441] that

$$
\operatorname{Ext}\left(B_{C(X)^{*}}\right)=\left\{\lambda e_{x}:(x, \lambda) \in X \times \mathbb{T}\right\}
$$

For $x \in X$ and $\lambda \in \mathbb{T}$, we define the map $\psi_{x, \lambda}: C(X)_{r} \rightarrow \mathbb{R}$ by $\psi_{x, \lambda}(f)=\operatorname{Re}(\lambda f(x))$ in fact, $\psi_{x, \lambda}=\operatorname{Re}\left(\lambda e_{x}\right)$. Clearly, $\psi_{x, \lambda} \in B_{\left(C(X)_{r}\right)^{*}}$ for all $(x, \lambda) \in X \times \mathbb{T}$. Kulkarni and Limaye showed [1, Proposition 3] that

$$
\operatorname{Ext}\left(B_{\left(C(X)_{r}\right)^{*}}\right)=\left\{\psi_{x, \lambda}:(x, \lambda) \in X \times \mathbb{T}\right\}
$$

and $\psi_{y, \mu}=\psi_{x, \lambda}$ if and only if $y=x$ and $\mu=\lambda$.

Let $\tau$ be a continuous involution on $X$; that is, $\tau: X \rightarrow X$ is continuous and $\tau \circ \tau$ is the identity map on $X$. The map $\sigma: C(X) \rightarrow C(X)$ defined by $\sigma(f)=\bar{f} \circ \tau$, is an algebra involution on $C(X)$ which is called the algebra involution induced by $\tau$ on $C(X)$. Define $C(X, \tau)=\{f \in C(X): \sigma(f)=f\}$. Then, $C(X, \tau)$ is a uniformly closed real subalgebra of $C(X)$ which contains 1 . The real algebras $C(X, \tau)$ were first considered in [3]. For a detailed account of several properties of $C(X, \tau)$, we refer to [4].

Let $P=\{(x, \lambda) \in X \times \mathbb{T}: \tau(x) \neq x\} \cup\{(x, \lambda) \in X \times \mathbb{T}: \tau(x)=x, \lambda \in\{-1,1\}\}$. For each $(x, \lambda) \in P$, let $\varphi_{x, \lambda}$ denote the restriction of $\psi_{x, \lambda}$ to $C(X, \tau)$. Grzesiak obtained a characterization of the extreme points of $B_{(C(X, \tau))^{*}}$ in [5] and showed that $\varphi \in \operatorname{Ext}\left(B_{\left.(C(X, \tau))^{*}\right)}\right.$ if and only if $\varphi=\varphi_{x, \lambda}$ for some $(x, \lambda) \in P$. Further, if $(x, \lambda),(y, \mu) \in P$, then $\varphi_{y, \mu}=\varphi_{x, \lambda}$ if and only if $(y, \mu)=(x, \lambda)$ or $(y, \mu)=(\tau(x), \bar{\lambda})$.

Kulkarni and Limaye obtained [1, Theorem 4] a characterization of $\operatorname{Ext}\left(B_{A^{*}}\right)$, where $A$ is a nonzero real linear subspace of $C(X, \tau)$. 
Let $(X, d)$ be a compact metric space. For $\alpha \in(0,1]$, we denote by $\operatorname{Lip}\left(X, d^{\alpha}\right)$ the set of all complex-valued functions $f$ on $X$ for which

$$
p_{\alpha}(f)=\sup \left\{\frac{|f(x)-f(y)|}{d^{\alpha}(x, y)}: x, y \in X, x \neq y\right\}
$$

is finite. Then, $\operatorname{Lip}(X, \alpha)$ is a complex subalgebra of $C(X)$ containing 1 and complex Banach space under the norm

$$
\|f\|_{X, p_{\alpha}}=\max \left\{\|f\|_{X^{\prime}} p_{\alpha}(f)\right\} \quad\left(f \in \operatorname{Lip}\left(X, d^{\alpha}\right)\right) .
$$

For $\alpha \in(0,1)$, the complex subalgebra of $\operatorname{Lip}\left(X, d^{\alpha}\right)$ consisting of all $f \in \operatorname{Lip}\left(X, d^{\alpha}\right)$ for which

$$
\frac{|f(x)-f(y)|}{d^{\alpha}(x, y)} \longrightarrow 0 \quad \text { as } d(x, y) \longrightarrow 0,
$$

is denoted by $\operatorname{lip}\left(X, d^{\alpha}\right)$. Clearly, $\operatorname{lip}\left(X, d^{\alpha}\right)$ is a closed linear subspace of $\left(\operatorname{Lip}\left(X, d^{\alpha}\right),\|\cdot\|_{X, p_{\alpha}}\right)$ and $1 \in \operatorname{lip}\left(X, d^{\alpha}\right)$. These Banach spaces were first studied by Leeuw in [6].

Given a compact metric space $(X, d)$, let $\tilde{X}=\{(x, y) \in X \times X: x \neq y\}$, and let the compact Hausdorff space $W$ be the disjoint union of $X$ with $\beta \tilde{X}$, where $\beta \tilde{X}$ is the Stone-Cech compactification of $\tilde{X}$. For $\alpha \in(0,1]$, consider the mapping $\Psi_{\alpha}: \operatorname{Lip}\left(X, d^{\alpha}\right) \rightarrow C(W)$ defined for each $f \in \operatorname{Lip}\left(X, d^{\alpha}\right)$ by

$$
\Psi_{\alpha}(f)(w)= \begin{cases}f(w) & \text { if } w \in X \\ (\beta \tilde{f})(w) & \text { if } w \in \beta \tilde{X}\end{cases}
$$

where

$$
\tilde{f}(x, y)=\frac{f(x)-f(y)}{d^{\alpha}(x, y)}, \quad \forall(x, y) \in \tilde{X},
$$

and $\beta \tilde{f}$ is the norm-preserving extension of $\tilde{f}$ to $\beta \tilde{X}$. Clearly, $\Psi_{\alpha}$ is a linear isometry from $\left(\operatorname{Lip}\left(X, d^{\alpha}\right),\|\cdot\|_{X, p_{\alpha}}\right)$ into $\left(C(W),\|\cdot\|_{W}\right)$, which is called the Leeuw's linear isometry. Therefore, $\Psi_{\alpha}\left(\operatorname{Lip}\left(X, d^{\alpha}\right)\right)$ is a uniformly closed linear subspace of $C(W)$. It is well known (see [2, page 441]) that

$$
\operatorname{Ext}\left(B_{\Psi_{\alpha}\left(\operatorname{Lip}\left(X, d^{\alpha}\right)\right)^{*}}\right) \subseteq\left\{\left.\lambda e_{w}\right|_{\Psi_{\alpha}\left(\operatorname{Lip}\left(X, d^{\alpha}\right)\right)}:(w, \lambda) \in W \times \mathbb{T}\right\},
$$

where $e_{w}$ is the evaluation functional at $w$ on $C(W)$.

For each $x \in X$ and $w \in W$, define the linear functionals $\Delta_{x}$ and $\widetilde{\Delta}_{w}$ in $\operatorname{Lip}\left(X, d^{\alpha}\right)^{*}$ by $\Delta_{x}(f)=f(x)$ and $\widetilde{\Delta}_{w}(f)=\Psi_{\alpha}(f)(w)$, respectively. Clearly, $\left|\Delta_{x}(f)\right| \leq\|f\|_{X, p_{\alpha}}$ and $\left|\widetilde{\Delta}_{w}(f)\right| \leq$ $\|f\|_{X, p_{\alpha}}$ for all $f \in \operatorname{Lip}\left(X, d^{\alpha}\right)$. Therefore, $\Delta_{x}, \widetilde{\Delta}_{w} \in B_{\operatorname{Lip}\left(X, d^{\alpha}\right)^{*}}$. Moreover, $\widetilde{\Delta}_{x}=\Delta_{x}$ for all $x \in X$ and $\tilde{\Delta}_{w}=e_{w} O \Psi_{\alpha}$ for all $w \in W$. Thus, we have the following result. 
Theorem 1.2. For $\alpha \in(0,1]$, every extreme point of $B_{\operatorname{Lip}\left(X, d^{\alpha}\right)^{*}}$ must be either of the form $\lambda \Delta_{x}$ with $(x, \lambda) \in X \times \mathbb{T}$ or of the form $\lambda \tilde{\Delta}_{w}$ with $(w, \lambda) \in \beta \tilde{X} \times \mathbb{T}$.

Roy proved the following result by using a result of Leeuw [6, Lemma 1.2].

Theorem 1.3 (see [7, Lemma 1.2]). For each $(x, \lambda) \in X \times \mathbb{T}, \lambda \Delta_{x}$ is an extreme point of $B_{\operatorname{Lip}\left(X, d^{\alpha}\right)^{*}}$.

Jimenez-Vargas and Villegas-Vallecillos used above results and obtained a characterization of linear isometries between $\operatorname{Lip}\left(X, d_{X}\right)$ and $\operatorname{Lip}\left(Y, d_{Y}\right)$ in [8].

A map $f: X \rightarrow Y$ is said to be Lipschitz map from the metric space $\left(X, d_{X}\right)$ to the metric space $\left(Y, d_{Y}\right)$ if there exists a constant $C>0$ such that $d_{Y}(f(x), f(y)) \leq C d_{X}(x, y)$ for all $x, y \in X$.

Let $(X, d)$ be a compact metric space. The mapping $\tau: X \rightarrow X$ is called a Lipschitz involution on $(X, d)$, if $\tau$ is a Lipschitz map from $(X, d)$ to itself and an involution on $X$. Clearly, every Lipschitz involution on $(X, d)$ is a continuous involution.

Let $\tau$ be a Lipschitz involution on the compact metric space $(X, d)$ and let $\sigma$ be the algebra involution induced by $\tau$ on $C(X)$. Clearly,

$$
\sigma\left(\operatorname{Lip}\left(X, d^{\alpha}\right)\right)=\operatorname{Lip}\left(X, d^{\alpha}\right), \quad \sigma\left(\operatorname{lip}\left(X, d^{\alpha}\right)\right)=\operatorname{lip}\left(X, d^{\alpha}\right)
$$

We define

$$
\begin{aligned}
\operatorname{Lip}\left(X, \tau, d^{\alpha}\right) & =\left\{h \in \operatorname{Lip}\left(X, d^{\alpha}\right): \sigma(h)=h\right\}, \\
\operatorname{lip}\left(X, \tau, d^{\alpha}\right) & =\left\{h \in \operatorname{lip}\left(X, d^{\alpha}\right): \sigma(h)=h\right\} .
\end{aligned}
$$

Then, the following statements hold.

(i) $\operatorname{Lip}\left(X, \tau, d^{\alpha}\right)\left(\operatorname{lip}\left(X, \tau, d^{\alpha}\right)\right.$, resp. $)$ is a real subalgebra of $\operatorname{Lip}\left(X, d^{\alpha}\right)\left(\operatorname{lip}\left(X, d^{\alpha}\right)\right.$, resp.).

(ii) $\operatorname{Lip}\left(X, \tau, d^{\alpha}\right)=\operatorname{Lip}\left(X, d^{\alpha}\right) \cap C(X, \tau)$ and $\operatorname{lip}\left(X, \tau, d^{\alpha}\right)=\operatorname{lip}\left(X, d^{\alpha}\right) \cap C(X, \tau)$.

(iii) $\operatorname{Lip}\left(X, d^{\alpha}\right)=\operatorname{Lip}\left(X, \tau, d^{\alpha}\right) \oplus i \operatorname{Lip}\left(X, \tau, d^{\alpha}\right)$ and $\operatorname{lip}\left(X, d^{\alpha}\right)=\operatorname{lip}\left(X, \tau, d^{\alpha}\right) \oplus i \operatorname{lip}(X$, $\left.\tau, d^{\alpha}\right)$.

(iv) $\operatorname{Lip}\left(X, \tau, d^{\alpha}\right)\left(\operatorname{lip}\left(X, \tau, d^{\alpha}\right)\right.$, resp.) is a real subalgebra of $C(X, \tau)$ which contains 1 and separates the points of $X$.

(v) $\operatorname{Lip}\left(X, \tau, d^{\alpha}\right)\left(\operatorname{lip}\left(X, \tau, d^{\alpha}\right)\right.$, resp.) is uniformly dense in $C(X, \tau)$ (use (iv) and the Stone-Weierstrass theorem for real subalgebra of $C(X, \tau)$ [3, Proposition 1.1].

(vi) For $0<\alpha<\beta \leq 1$,

$$
\operatorname{Lip}\left(X, \tau, d^{\beta}\right) \subseteq \operatorname{lip}\left(X, \tau, d^{\alpha}\right) \subseteq \operatorname{Lip}\left(X, \tau, d^{\alpha}\right) .
$$

(vii) There exists a constant $C \geq 1$ such that

$$
\max \left\{\|f\|_{X, p_{\alpha^{\prime}}}\|g\|_{X, p_{\alpha}}\right\} \leq C^{\alpha}\|f+g\|_{X, p_{\alpha^{\prime}}}
$$

for all $f, g \in \operatorname{Lip}\left(X, \tau, d^{\alpha}\right)$.

(viii) $\left.\operatorname{Lip}\left(X, \tau, d^{\alpha}\right),\|\cdot\|_{X, p_{\alpha}}\right)$ is a real Banach space and $\operatorname{lip}\left(X, \tau, d^{\alpha}\right)$ is its closed real subspace. 
The real Banach spaces $\operatorname{Lip}\left(X, \tau, d^{\alpha}\right)$ and $\operatorname{lip}\left(X, \tau, d^{\alpha}\right)$ are called real Banach spaces of complex Lipschitz functions and first studied in [9].

We give a characterization of extreme points of the unit ball in the dual space of $\operatorname{Lip}\left(X, d^{\alpha}\right)_{r}, \operatorname{Lip}\left(X, \tau, d^{\alpha}\right)$ and some its real linear subspaces for $\alpha \in(0,1]$ in Section 2. Next, we give a characterization of extreme points of the unit ball in the dual spaces of $\operatorname{lip}\left(X, d^{\alpha}\right), \operatorname{lip}\left(X, d^{\alpha}\right)_{r}, \operatorname{lip}\left(X, \tau, d^{\alpha}\right)$ and some its real linear subspaces for $\alpha \in(0,1)$ in Section 3.

\section{Real Linear Subspaces of $\operatorname{Lip}\left(X, d^{\alpha}\right)$ Containing 1}

In the remainder of this paper, we assume that $\alpha \in(0,1],(X, d)$ is a compact metric space, $\tilde{X}=\{(x, y) \in X \times X, x \neq y\}, \beta \tilde{X}$ is the Stone-Cech compactification of $\tilde{X}, W$ is the compact Hausdorff space $X \cup \beta \tilde{X}, \Psi_{\alpha}$ is the Leeuw's linear isometry from $\left(\operatorname{Lip}\left(X, d^{\alpha}\right),\|\cdot\|_{X, p_{\alpha}}\right)$ into $\left(C(W),\|\cdot\|_{W}\right)$, and $\tau$ is a Lipschitz involution on $(X, d)$.

For each $(w, \lambda) \in W \times \mathbb{T}$, we define the map $\widetilde{\psi}_{w, \lambda}: \operatorname{Lip}\left(X, d^{\alpha}\right)_{r} \rightarrow \mathbb{R}$ by $\widetilde{\psi}_{w, \lambda}(f)=$ $\operatorname{Re}\left(\lambda \widetilde{\Delta}_{w}(f)\right)$ in fact, $\widetilde{\psi}_{w, \lambda}=\operatorname{Re}\left(\lambda\left(e_{w} o \Psi_{\alpha}\right)\right)$. Clearly, $\widetilde{\psi}_{w, \lambda} \in B_{\left(\operatorname{Lip}\left(X, d^{\alpha}\right)_{r}\right)^{*}}$ for all $(w, \lambda) \in W \times \mathbb{T}$. Moreover, $\widetilde{\psi}_{x, \lambda}=\operatorname{Re}\left(\lambda \Delta_{x}\right)$ for all $(x, \lambda) \in X \times \mathbb{T}$.

We first give a characterization of the extreme points of the unit ball in the $\left(\operatorname{Lip}\left(X, d^{\alpha}\right)_{r}\right)^{*}$ as the following.

Proposition 2.1. By above notations,

$$
\left\{\tilde{\psi}_{x, \lambda}:(x, \lambda) \in X \times \mathbb{T}\right\} \subseteq \operatorname{Ext}\left(B_{\left(\operatorname{Lip}\left(X, d^{\alpha}\right)_{r}\right)^{*}}\right) \subseteq\left\{\tilde{\psi}_{w, \lambda}:(w, \lambda) \in W \times \mathbb{T}\right\}
$$

Further, for $(x, \lambda),(y, \mu) \in X \times \mathbb{T}$ one has $\tilde{\psi}_{x, \lambda}=\widetilde{\psi}_{y, \mu}$ if and only if $(x, \lambda)=(y, \mu)$.

Proof. We define the map $T:\left(\operatorname{Lip}\left(X, d^{\alpha}\right)^{*}\right)_{r} \rightarrow\left(\operatorname{Lip}\left(X, d^{\alpha}\right)_{r}\right)^{*}$ by $T(\varphi)=\operatorname{Re} \varphi$. Clearly, $T$ is a real-linear mapping. For each $u \in\left(\operatorname{Lip}\left(X, d^{\alpha}\right)_{r}\right)^{*}$, defining the map $\varphi: \operatorname{Lip}\left(X, d^{\alpha}\right)^{*} \rightarrow \mathbb{C}$ by $\varphi(f)=u(f)-i u(i f)$. Clearly, $\varphi \in \operatorname{Lip}\left(X, d^{\alpha}\right)^{*}$ and $u=\operatorname{Re} \varphi$. It follows that $\varphi \in\left(\operatorname{Lip}\left(X, d^{\alpha}\right)^{*}\right)_{r}$ and $T(\varphi)=u$. Thus, $T$ is onto.

We claim that $T$ is an isometric. Let $\varphi \in\left(\operatorname{Lip}\left(X, d^{\alpha}\right)^{*}\right)_{r}$. Since

$$
|T(\varphi)(f)|=|(\operatorname{Re} \varphi)(f)|=|\operatorname{Re}(\varphi(f))| \leq|\varphi(f)| \leq\|\varphi\|\|f\|_{X, p_{\alpha^{\prime}}}
$$

for each $f \in \operatorname{Lip}\left(X, d^{\alpha}\right)_{r}$, we have

$$
\|T(\varphi)\| \leq\|\varphi\|
$$

Let $\varepsilon$ be an arbitrary positive number. There exists $f \in \operatorname{Lip}\left(X, d^{\alpha}\right)_{r}$ with $\|f\|_{X, p_{\alpha}} \leq 1$ such that $\|\varphi\|<|\psi(f)|+\varepsilon$. Choose $\gamma=1$ if $\psi(f)=0$ and $\gamma=(1 / \psi(f))|\varphi(f)|$ if $\psi(f) \neq 0$. Then, $\gamma \in \mathbb{C},|\gamma|=1$ and $\psi(f)=\gamma|\psi(f)|$. If $g=(1 / \gamma) f$, then $g \in \operatorname{Lip}\left(X, d^{\alpha}\right)_{r},\|g\|_{X, p_{\alpha}}=\|f\|_{X, p_{\alpha}} \leq 1$ and so,

$$
\|\psi\|<|\operatorname{Re}(\varphi(g))|+\varepsilon=|T(\varphi)(g)|+\varepsilon \leq\|T(\varphi)\|+\varepsilon .
$$


It follows that

$$
\|\varphi\| \leq\|T(\varphi)\|
$$

Thus, our claim is justified. The above arguments show that $T$ is a real-linear isometry from $\left(\operatorname{Lip}\left(X, d^{\alpha}\right)^{*}\right)_{r}$ onto $\left(\operatorname{Lip}\left(X, d^{\alpha}\right)_{r}\right)^{*}$. Therefore,

$$
\operatorname{Ext}\left(B_{\left(\operatorname{Lip}\left(X, d^{\alpha}\right)_{r}\right)^{*}}\right)=T\left(\operatorname{Ext}\left(B_{\operatorname{Lip}\left(x, d^{\alpha}\right)^{*}}\right)\right) .
$$

Since

$$
\left\{\lambda \widetilde{\Delta}_{x}:(x, \lambda) \in X \times \mathbb{T}\right\} \subseteq \operatorname{Ext}\left(B_{\operatorname{Lip}\left(X, d^{\alpha}\right)^{*}}\right) \subseteq\left\{\lambda \tilde{\Delta}_{w}:(w, \lambda) \in W \times \mathbb{T}\right\}
$$

we conclude that

$$
\left\{T\left(\lambda \widetilde{\Delta}_{x}\right):(x, \lambda) \in X \times \mathbb{T}\right\} \subseteq \operatorname{Ext}\left(B_{\left(\operatorname{Lip}\left(X, d^{\alpha}\right)_{r}\right)^{*}}\right) \subseteq\left\{T\left(\lambda \tilde{\Delta}_{w}\right):(w, \lambda) \in W \times \mathbb{T}\right\},
$$

by Theorems 1.2 and 1.3 .

Clearly, $T\left(\lambda \widetilde{\Delta}_{w}\right)=\widetilde{\Psi}_{\lambda, w}$ for all $(w, \lambda) \in W \times \mathbb{T}$. Therefore,

$$
\left\{\widetilde{\Psi}_{\lambda, x}:(x, \lambda) \in X \times \mathbb{T}\right\} \subseteq \operatorname{Ext}\left(B_{\left(\operatorname{Lip}\left(X, d^{\alpha}\right)_{r}\right)^{*}}\right) \subseteq\left\{\widetilde{\Psi}_{\lambda, w}:(w, \lambda) \in W \times \mathbb{T}\right\}
$$

It is obvious that if $(x, \lambda),(y, \mu) \in X \times \mathbb{T}$ and $(x, \lambda)=(y, \mu)$, then $\widetilde{\Psi}_{\lambda, x}=\widetilde{\Psi}_{\mu, y}$. We now assume that $\widetilde{\Psi}_{\lambda, x}=\widetilde{\Psi}_{\mu, y}$, where $(x, \lambda),(y, \mu) \in X \times \mathbb{T}$. Letting $f=1$ and $f=i$, we see that $\operatorname{Re} \lambda=\operatorname{Re} \mu$ and $\operatorname{Re}(\lambda i)=\operatorname{Re}(\mu i)$; that is, $\lambda=\mu$. If $x \neq y$, there exists $f \in \operatorname{Lip}\left(X, d^{\alpha}\right)_{r}$ such that $f(y)=\bar{\mu}$, but $f(x)=0$ (define $f: X \rightarrow \mathbb{C}$ by $f(z)=(\bar{\mu} / d(y, x)) d(z, x), z \in X)$; so that

$$
|\mu|^{2}=\operatorname{Re}(\mu \bar{\mu})=\operatorname{Re}(\mu f(y))=\widetilde{\Psi}_{y, \mu}(f)=\widetilde{\Psi}_{x, \lambda}(f)=\operatorname{Re}(\lambda f(x))=0 .
$$

But this is not possible since $|\mu|=1$. Thus, $x=y$.

The next purpose is giving conditions for $\varphi \in B_{A^{*}}$ to be an extreme point of $B_{A^{*}}$, where $A$ is a real subspace of $\operatorname{Lip}\left(X, \tau, d^{\alpha}\right)$.

Theorem 2.2. Let $A$ be a real linear subspace of $\operatorname{Lip}\left(X, \tau, d^{\alpha}\right)$ containing 1 . For $(w, \lambda) \in W \times \mathbb{T}$, let $\tilde{\eta}_{w, \lambda}=\left.\tilde{\psi}_{w, \lambda}\right|_{A}$. Let $P=\{(x, \lambda) \in X \times \mathbb{T}: \tau(x) \neq x\} \cup\{(x, \lambda) \in X \times\{-1,1\}: \tau(x)=x\} \cup\{(w, \lambda)$ : $w \in \beta \tilde{X}, \lambda \in \mathbb{T}\}$.

Let $Q_{A}$ denote the set of $(x, \lambda) \in X \times\{-1,1\}$ such that

(i) there is $g \in A$ with $\|g\|_{X, p_{\alpha}} \leq 1$ and $g(x)=\lambda$,

(ii) for every $y \in X \backslash\{x, \tau(x)\}$, there is some $h \in A$ with $\|h\|_{X, p_{\alpha}} \leq 1, h(x)=1$ and $|h(y)|<1$.

Then,

$$
\left\{\tilde{\eta}_{x, \lambda}:(x, \lambda) \in Q_{A}\right\} \subseteq \operatorname{Ext}\left(B_{A^{*}}\right) \subseteq\left\{\tilde{\eta}_{w, \lambda}:(w, \lambda) \in P\right\}
$$


Further, if $(x, \lambda) \in Q_{A}$ and $(y, \mu) \in P \cap(X \times \mathbb{T})$, then $\tilde{\eta}_{y, \mu}=\tilde{\eta}_{x, \lambda}$ if and only if either $(y, \mu)=(x, \lambda)$ or $(y, \mu)=(\tau(x), \lambda)$.

Proof. Let $\varphi \in \operatorname{Ext}\left(B_{A^{*}}\right)$. Letting $\mathfrak{X}=\operatorname{Lip}\left(X, d^{\alpha}\right)_{r}$ in part (a) of Theorem 1.1, and using Proposition 2.1, we see that $\varphi=\tilde{\eta}_{w, \lambda}$ for some $(w, \lambda) \in W \times \mathbb{T}$. To prove that $(w, \lambda) \in P$, we consider $w=x \in X$ with $\tau(x)=x$ and show that $\lambda \in\{-1,1\}$. For every $f \in A$, we have $\overline{f(x)}=f(\tau(x))=f(x)$ that is, $f(x)$ is a real number. Hence,

$$
|\varphi(f)|=\left|\tilde{\eta}_{x, \lambda}(f)\right|=\left|\tilde{\psi}_{x, \lambda}(f)\right|=|\operatorname{Re}(\lambda f(x))|=|\operatorname{Re} \lambda||f(x)| \leq|\operatorname{Re} \lambda|\|f\|_{X, p_{\alpha}} .
$$

This shows that $\|\varphi\| \leq|\operatorname{Re} \lambda|$. But since $\varphi$ is an extreme point of $B_{A^{*}}$, we must have $\|\varphi\|=1$. Thus, $1 \leq|\operatorname{Re} \lambda| \leq|\lambda|=1$ so that $\lambda \in\{-1,1\}$.

Next, let $(x, \lambda) \in Q_{A}$. We claim that the following statement hold.

For $(y, \mu) \in X \times \mathbb{T}$ with $(y, \mu) \neq(x, \lambda)$ and $(y, \mu) \neq(\tau(x), \lambda)$, there is $f \in A$ such that $\|f\|_{X, p_{\alpha}}=1, \operatorname{Re}(\lambda f(x))=1$, but $\operatorname{Re}(\mu f(y)) \neq 1$.

By condition (i), there is $g \in A$ such that $\|g\|_{X, p_{\alpha}} \leq 1$ and $g(x)=\lambda$.

Case $1(y \in\{x, \tau(x)\})$. Let $f=g$. Then, $\|f\|_{X, p_{\alpha}} \leq 1$ and $|f(x)|=|\bar{\lambda}|=1$ so that $\|f\|_{X, p_{\alpha}}=1$. Also,

$$
\operatorname{Re}(\lambda f(x))=\operatorname{Re}(\lambda g(x))=\operatorname{Re}\left(\lambda^{2}\right)=1
$$

If $y=x$, then $(y, \mu) \neq(x, \lambda)$ implies that $\mu \neq \lambda$ that is, $\mu \lambda \neq 1$. Since $|\mu \lambda|=1$, this shows that

$$
\operatorname{Re}(\mu f(x))=\operatorname{Re}(\mu g(x))=\operatorname{Re}(\mu \lambda) \neq 1
$$

If $y=\tau(x)$, then $(y, \mu) \neq(\tau(x), \lambda)$ implies that $\mu \neq \lambda$, that is, $\mu \lambda \neq 1$. Since $|\mu \lambda|=1$, this shows that

$$
\operatorname{Re}(\mu f(y))=\operatorname{Re}(\mu g(\tau(x)))=\operatorname{Re}(\mu \bar{\lambda})=\operatorname{Re}(\mu \lambda) \neq 1
$$

Case $2(y \notin\{x, \tau(x)\})$. By condition (ii), there is $h \in A$ such that $\|h\|_{X, p_{\alpha}} \leq 1, h(x)=1$ and $|h(y)|<1$. Let $f=g h$. Now, $\|f\|_{X, p_{\alpha}} \leq 1$ and $|f(x)|=|f(x) \| g(x)|=|g(x)|=|\lambda|=1$, so that $\|f\|_{X, p_{\alpha}}=1$. Also,

$$
\begin{aligned}
\operatorname{Re}(\lambda f(x)) & =\operatorname{Re}(\lambda g(x) h(x))=\operatorname{Re}\left(\lambda^{2}\right)=1, \\
\operatorname{Re}(\mu f(y)) & =\operatorname{Re}(\mu g(y) h(y)) \leq|\mu g(y) h(y)| \\
& =|g(y)||h(y)| \leq|h(y)|<1 .
\end{aligned}
$$

Thus, our claim is justified. Let $\mathfrak{X}=\operatorname{Lip}\left(X, d^{\alpha}\right)_{r}$ and $\varphi=\tilde{\eta}_{x, \lambda}$ in part (b) of Theorem 1.1. Consider $\psi \in \operatorname{Ext}\left(B_{\mathfrak{X}^{*}}\right)$ such that $\psi(f)=1$ for all $f \in A$ with $\varphi(f)=1=\|f\|_{X, p_{\alpha}}$. By Proposition 2.1, $\psi=\widetilde{\psi}_{w, \mu}$ for some $(w, \mu) \in W \times \mathbb{T}$. Thus, $\operatorname{Re}\left(\mu \widetilde{\Delta}_{w}(f)\right)=1$ for all $f \in A$ 
with $\operatorname{Re}(\lambda f(x))=1=\|f\|_{X, p_{\alpha}}$. Since $\lambda 1 \in A,\|\lambda 1\|_{X, p_{\alpha}}=1$ and $\operatorname{Re}(\lambda(\lambda 1)(x))=\operatorname{Re}\left(\lambda^{2}\right)=1$, we have $\operatorname{Re}\left(\mu \psi_{\alpha}(\lambda 1)(w)\right)=1$. If $w^{\prime} \in \beta \tilde{X}$, then

$$
\begin{aligned}
\operatorname{Re}\left(\mu \psi_{\alpha}(\lambda 1)\left(w^{\prime}\right)\right) & =\operatorname{Re}\left(\mu \lambda \psi_{\alpha}(1)\left(w^{\prime}\right)\right)=\operatorname{Re}\left(\mu \lambda(\beta \tilde{1})\left(w^{\prime}\right)\right) \\
& \leq\left|\left(\mu \lambda(\beta \tilde{1})\left(w^{\prime}\right)\right)\right|=\left|(\beta \tilde{1})\left(w^{\prime}\right)\right| \\
& \leq\|\beta \tilde{1}\|_{\beta \tilde{X}}=\|\tilde{1}\|_{\tilde{X}} \\
& =p_{\alpha}(1)=0 .
\end{aligned}
$$

Thus, it must be $w \in X$. Choose $w=y$. Then, $(y, \mu) \in X \times \mathbb{T}$ and $\operatorname{Re}(\mu f(y))=1$ for all $f \in A$ with $\|f\|_{X, p_{\alpha}}=1=\operatorname{Re}(\lambda f(x))$. By our claim, we must have $(y, \mu)=(x, \lambda)$ or $(y, \mu)=(\tau(x), \lambda)$. If $(y, \mu)=(x, \lambda)$, then clearly

$$
\left.\psi\right|_{A}=\left.\widetilde{\varphi}_{y, \mu}\right|_{A}=\left.\tilde{\varphi}_{x, \lambda}\right|_{A}=\tilde{\eta}_{x, \lambda}=\varphi
$$

Now, let $(y, \mu)=(\tau(x), \lambda)$. Since $\lambda \in \mathbb{R}, f \in A$ implies that

$$
\begin{aligned}
\psi(f) & =\operatorname{Re}(\mu f(y))=\operatorname{Re}(\lambda f(\tau(x))) \\
& =\operatorname{Re}(\lambda \bar{f}(x))=\lambda \operatorname{Re}(\overline{f(x)})=\lambda \operatorname{Re}(f(x)) \\
& =\operatorname{Re}(\lambda f(x))=\widetilde{\psi}_{x, \lambda}(f) \\
& =\tilde{\eta}_{x, \lambda}(f)=\varphi(f) .
\end{aligned}
$$

Therefore, $\left.\psi\right|_{A}=\varphi$, again. Hence, we see that $\tilde{\eta}_{(x, \lambda)}=\varphi \in \operatorname{Ext}\left(B_{A^{*}}\right)$. This also shows that $\tilde{\eta}_{\tau(x), \lambda}=\tilde{\eta}_{x, \lambda}$ for all $(x, \lambda) \in X \times\{-1,1\}$.

Conversely, our claim implies that if $(x, \lambda) \in Q_{A},(y, \mu) \in X \times \mathbb{T}$ and $\tilde{\eta}_{y, \mu}=\tilde{\eta}_{x, \lambda}$, then $(y, \mu)=(x, \lambda)$ or $(y, \mu)=(\tau(x), \lambda)$. Thus, for $(x, \lambda) \in Q_{A}$ and $(y, \mu) \in P \cap(X \times \mathbb{T})$, we have $\tilde{\eta}_{y, \mu}=\tilde{\eta}_{x, \lambda}$ if and only if $(y, \mu)=(x, \lambda)$ or $(y, \mu)=(\tau(x), \lambda)$.

By using the above theorem, we give a characterization of extreme points of unit ball in the dual space of $\operatorname{Lip}\left(X, \tau, d^{\alpha}\right)$.

Corollary 2.3. Let $P$ be as in Theorem 2.2. For $(w, \lambda) \in P$, let $\tilde{\eta}_{w, \lambda}$ denote the restriction of $\tilde{\psi}_{w, \lambda}$ to $\operatorname{Lip}\left(X, \tau, d^{\alpha}\right)$. Then,

$$
\left\{\tilde{\eta}_{x, \lambda}:(x, \lambda) \in X \times\{-1,1\}\right\} \subseteq \operatorname{Ext}\left(B_{\operatorname{Lip}\left(X, \tau, d^{\alpha}\right)^{*}}\right) \subseteq\left\{\tilde{\eta}_{w, \lambda}:(w, \lambda) \in P\right\}
$$

Further, if $(x, \lambda) \in X \times\{-1,1\}$ and $(y, \mu) \in P \cap(X \times \mathbb{T})$, then $\eta_{y, \mu}=\tilde{\eta}_{x, \lambda}$ if and only if $(y, \mu)=(x, \lambda)$ or $(y, \mu)=(\tau(x), \lambda)$. 
Proof. Let $A=\operatorname{Lip}\left(X, \tau, d^{\alpha}\right)$. By Theorem 2.2, we have

$$
\operatorname{Ext}\left(B_{A^{*}}\right) \subseteq\left\{\tilde{\eta}_{w, \lambda}:(w, \lambda) \in P\right\}
$$

To prove $\left\{\tilde{\eta}_{x, \lambda}:(x, \lambda) \in X \times\{-1,1\}\right\} \subseteq \operatorname{Ext}\left(B_{\operatorname{Lip}\left(X, \tau, d^{\alpha}\right)^{*}}\right)$, it is enough to show that for every $(x, \lambda) \in X \times\{-1,1\}$,

(i) there is $g \in \operatorname{Lip}\left(X, \tau, d^{\alpha}\right)$ with $\|g\|_{X, p_{\alpha}} \leq 1$ and $g(x)=\lambda$,

(ii) for every $y \in x \backslash\{x, \tau(x)\}$, there is $h \in \operatorname{Lip}\left(X, \tau, d^{\alpha}\right)$ with

$$
\|h\|_{X, p_{\alpha}} \leq 1, \quad h(x)=1, \quad|h(y)|<1 .
$$

Let $(x, \lambda) \in X \times\{-1,1\}$. We first define the function $g: X \rightarrow \mathbb{C}$ by

$$
g(z)=\lambda\left(1-\frac{d(z, x) d(\tau(z), x)}{1+\rho^{2}+\rho^{2-2 \alpha}}\right)
$$

where $\rho=\operatorname{diam}(X)=\sup \{d(y, z): y, z \in X\}$. Clearly, $g \in C(X, \tau), g(x)=\lambda,\|g\|_{X} \leq 1$ and $g \in \operatorname{Lip}\left(X, d^{1}\right)$. Let $y, z \in X$ with $y \neq z$. Then,

$$
\begin{aligned}
\frac{|g(y)-g(z)|}{d^{\alpha}(y, z)} & =\frac{|d(z, x) d(\tau(z), x)-d(y, x) d(\tau(y), x)|}{\left(1+\rho^{2}+\rho^{2-2 \alpha}\right) d^{\alpha}(y, z)} \\
& \leq \frac{d^{1-\alpha}(y, z)}{\left(1+\rho^{2}+\rho^{2-2 \alpha}\right)}(d(z, x)+d(\tau(y), x)) \\
& \leq \frac{2 \rho \rho^{1-\alpha}}{1+\rho^{2}+\rho^{2-2 \alpha}} \\
& \leq 1 .
\end{aligned}
$$

Therefore, $g \in \operatorname{Lip}\left(X, d^{\alpha}\right)$ and $p_{\alpha}(g) \leq 1$. Consequently,

$$
g \in \operatorname{Lip}\left(X, \tau, d^{\alpha}\right), \quad\|g\|_{X, p_{\alpha}} \leq 1, \quad g(x)=\lambda .
$$

Hence, (i) holds.

We now assume that $y \in X \backslash\{x, \tau(x)\}$ and define the function $h: X \rightarrow \mathbb{C}$ by

$$
h(z)=1-\frac{\lambda g(z) d(x, z) d(x, \tau(z))}{2\left(1+\rho^{2}+\rho^{2-2 \alpha}\right)} .
$$

Clearly, $h \in C(X, \tau), h(x)=1,\|h\|_{X} \leq 1$ and there exists $\gamma \in(0,1]$ such that $h(y)=1-\gamma$ so that $|h(y)|<1$. We define the complex-valued function $g_{1}$ on $X$ by $g_{1}=(1 / 2)(1-\lambda g)$. Then, 
$g_{1} \in C(X, \tau),\left\|g_{1}\right\|_{X} \leq 1 / 2, g_{1} \in \operatorname{Lip}\left(X, d^{1}\right), p_{\alpha}\left(g_{1}\right) \leq 1 / 2$ and $h=1-g g_{1}$. Thus, we have $h \in \operatorname{Lip}\left(X, d^{1}\right), h \in \operatorname{Lip}\left(X, \tau, d^{\alpha}\right)$ and

$$
p_{\alpha}(h) \leq p_{\alpha}(g)\left\|g_{1}\right\|_{X}+\|g\|_{X} p_{\alpha}\left(g_{1}\right) \leq \frac{1}{2}+\frac{1}{2}=1,
$$

so that $\|h\|_{X, p_{\alpha}} \leq 1$. Since $y \in X \backslash\{x, \tau(x)\},|h(y)|=h(y)<1$. Thus, (ii) holds.

\section{Real Subspaces of $\operatorname{lip}\left(X, d^{\alpha}\right)$ Containing 1}

Throughout this section, we assume that $\alpha \in(0,1)$. Consider the mapping $\Phi_{\alpha}: \operatorname{lip}\left(X, d^{\alpha}\right) \rightarrow$ $C(W)$ by $\Phi_{\alpha}=\left.\Psi_{\alpha}\right|_{\operatorname{lip}\left(X, d^{\alpha}\right)}$. Then, $\Phi_{\alpha}$ is a linear isometric from $\left(\operatorname{lip}\left(X, d^{\alpha}\right),\|\cdot\|_{X, p_{\alpha}}\right)$ into $C(W)$.

For each $x \in X$ and $w \in W$, define the functionals $\delta_{x}$ and $\widetilde{\delta}_{w}$ in $\operatorname{lip}\left(X, d^{\alpha}\right)^{*}$ by $\delta_{x}(f)=$ $f(x)$ and $\tilde{\delta}_{w}(f)=\Phi_{\alpha}(f)(w)$, respectively. Clearly, $\left|\delta_{x}(f)\right| \leq\|f\|_{X, p_{\alpha}}$ for all $f \in \operatorname{lip}\left(X, d^{\alpha}\right)$ and $\left|\widetilde{\delta}_{w}(f)\right| \leq\|f\|_{X, p_{\alpha}}$, and therefore, $\delta_{x}, \widetilde{\delta}_{w} \in B_{\operatorname{lip}\left(X, d^{\alpha}\right)^{*}}$. Moreover, $\widetilde{\delta}_{x}=\delta_{x}$ for all $x \in X$ and $\tilde{\delta}_{w}=e_{w} O \Phi_{\alpha}$ for all $w \in W$.

We give a characterization of extreme points of the unit ball in the dual space $\operatorname{lip}\left(X, d^{\alpha}\right)$ as the following.

Theorem 3.1. Every extreme point of $B_{\operatorname{lip}\left(X, d^{\alpha}\right)^{*}}$ must be either the form $\lambda \delta_{x}$ with $(x, \lambda) \in X \times \mathbb{T}$ or of the form $\lambda \tilde{\delta}_{w}$ with $(\lambda, w) \in \beta \tilde{X} \times \mathbb{T}$. Moreover, $\lambda \delta_{x}$ is an extreme point of $B_{\operatorname{lip}\left(X, d^{\alpha}\right)^{*}}$ for all $(x, \lambda) \in X \times \mathbb{T}$.

Proof. Since $\left(\operatorname{lip}\left(X, d^{\alpha}\right),\|\cdot\|_{X, p_{\alpha}}\right)$ is a Banach space and $\Phi_{\alpha}$ is a linear isometry from $\left(\operatorname{lip}\left(X, d^{\alpha}\right),\|\cdot\|_{X, p_{\alpha}}\right)$ into $\left(C(W),\|\cdot\|_{W}\right)$, we conclude that $\Phi_{\alpha}\left(\operatorname{lip}\left(X, d^{\alpha}\right)\right)$ is a uniformly closed subspace of $C(W)$. It is well known [2, page 441] that

$$
\operatorname{Ext}\left(B_{\left(\Phi_{\alpha}\left(\operatorname{lip}\left(X, d^{\alpha}\right)\right)\right)^{*}}\right) \subseteq\left\{\left.\lambda e_{w}\right|_{\Phi_{\alpha}\left(\operatorname{lip}\left(X, d^{\alpha}\right)\right)}:(w, \lambda) \in W \times \mathbb{T}\right\}
$$

Let $A=\Phi_{\alpha}\left(\operatorname{lip}\left(X, d^{\alpha}\right)\right)$ and define $\Lambda_{\alpha}: \operatorname{lip}\left(X, d^{\alpha}\right) \rightarrow A$ by $\Lambda_{\alpha}(f)=\Phi_{\alpha}(f)$. Then, $\Lambda_{\alpha}$ is a linear isometry from $\left(\operatorname{lip}\left(X, d^{\alpha}\right),\|\cdot\|_{p_{\alpha}}\right)$ onto $\left(A,\|\cdot\|_{W}\right)$, so $\Lambda_{\alpha}^{*}$, the adjoint of $\Lambda_{\alpha}$, is a linear isometry from $\left(A,\|\cdot\|_{W}\right)^{*}$ onto $\left(\operatorname{lip}\left(X, d^{\alpha}\right)\right)^{*}$. It is easily to show that $\Lambda_{\alpha}^{*}\left(\left.e_{w}\right|_{A}\right)=\widetilde{\delta}_{w}$ for all $w \in W$. Let

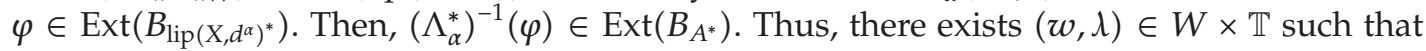
$\left(\Lambda_{\alpha}^{*}\right)^{-1}(\varphi)=\left.\lambda e_{w}\right|_{A}$ so that $\varphi=\lambda_{\alpha}^{*}\left(\left.e_{w}\right|_{A}\right)=\lambda \widetilde{\delta}_{w}$. It follows that

$$
\operatorname{Ext}\left(B_{\operatorname{lip}\left(X, d^{\alpha}\right)^{*}}\right) \subseteq\left\{\lambda \tilde{\delta}_{w}:(w, \lambda) \in W \times \mathbb{T}\right\}
$$

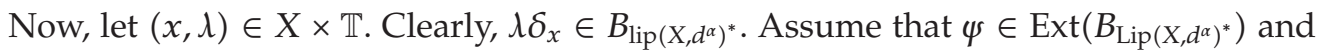
$\psi(f)=1$ for all $f \in \operatorname{lip}\left(X, d^{\alpha}\right)$ with $\left(\lambda \delta_{x}\right)(f)=1=\|f\|_{X, p_{\alpha}}$. Since $\operatorname{lip}\left(X, d^{\alpha}\right)$ is a nonzero linear subspace of $\operatorname{Lip}\left(X, d^{\alpha}\right)$, we conclude that $\psi=\mu \Delta_{y}$ for some $(y, \mu) \in X \times \mathbb{T}$ or $\psi=\mu \widetilde{\Delta}_{w}$ for 
some $(w, \mu) \in(\beta \tilde{X}) \times \mathbb{T}$ by Theorem 1.1. Clearly, $\bar{\lambda} 1 \in \operatorname{lip}\left(X, d^{\alpha}\right)$ and $\left(\lambda \delta_{x}\right)(\bar{\lambda} 1)=1=\|\bar{\lambda} 1\|_{X, p_{\alpha}}$. If $\psi=\mu \widetilde{\Delta}_{w}$ for some $(w, \mu) \in(\beta \tilde{X}) \times \mathbb{T}$, then we have

$$
\begin{aligned}
1 & =|\psi(\bar{\lambda} 1)|=\left|\mu \widetilde{\Delta}_{w}(\bar{\lambda} 1)\right| \\
& =\left|\widetilde{\Delta}_{w}(1)\right|=|(\beta \tilde{1})(w)| \\
& \leq\|\beta \tilde{1}\|_{\beta \tilde{\mathrm{X}}}=p_{\alpha}(1) \\
& =0 .
\end{aligned}
$$

Thus, it must be $\psi=\mu \Delta_{y}$ for some $(y, \mu) \in X \times \mathbb{T}$. Since $\psi(\bar{\lambda} 1)=1$, we have

$$
1=\left(\mu \Delta_{y}\right)(\bar{\lambda} 1)=\mu \bar{\lambda}
$$

It follows that $\mu=\lambda$. We claim that $y=x$. Let $y \in X \backslash\{x\}$. We define the function $g: X \rightarrow \mathbb{C}$ by

$$
g(z)=1-\frac{d(z, x)}{1+\rho+\rho^{1-\alpha}}
$$

where $\rho=\operatorname{diam} X$. It is easy to show that $g \in \operatorname{Lip}\left(X, d^{1}\right), g(x)=1,\|g\|_{X}=1$ and $p_{\alpha}(g) \leq 1$. Now, we define the function $f: X \rightarrow \mathbb{C}$ by

$$
f(z)=\bar{\lambda}\left(1-\frac{g(z) d(z, x)}{2\left(1+\rho+\rho^{1-\alpha}\right)}\right),
$$

and the function $g_{1}: X \rightarrow \mathbb{C}$ by $g_{1}=(1 / 2)(1-g)$. It is easy to see that $g_{1} \in \operatorname{Lip}\left(X, d^{1}\right)$, $\left\|g_{1}\right\|_{X} \leq 1 / 2, p_{\alpha}\left(g_{1}\right) \leq 1 / 2$, and $f=\bar{\lambda}\left(1-g g_{1}\right)$. Therefore, we have $f \in \operatorname{Lip}\left(X, d^{1}\right) \subseteq \operatorname{lip}\left(X, d^{\alpha}\right)$, $f(x)=\bar{\lambda},\|f\|_{X} \leq 1$, and

$$
p_{\alpha}(f) \leq p_{\alpha}(g)\left\|g_{1}\right\|_{X}+\|g\|_{X} p_{\alpha}\left(g_{1}\right) \leq \frac{1}{2}+\frac{1}{2}=1
$$

so that $\|f\|_{X, p}=1=\left(\lambda \delta_{x}\right)(f)$. It must be that $\psi(f)=1$; that is, $\left(\lambda \Delta_{y}\right)(f)=1$. But

$$
\begin{aligned}
\left|\left(\lambda \Delta_{y}\right)(f)\right| & =|\lambda f(y)|=|f(y)| \\
& =1-\frac{g(y) d(y, x)}{2\left(1+\rho+\rho^{1-\alpha}\right)}<1 .
\end{aligned}
$$


This contradiction implies that $y=x$ and so our claim is justified. Therefore, $\psi=\lambda \Delta_{x}$. It follows that $\left.\psi\right|_{\operatorname{lip}\left(X, d^{\alpha}\right)}=\lambda \delta_{x}$. Therefore, $\lambda \delta_{x} \in \operatorname{Ext}\left(B_{\operatorname{lip}\left(X, d^{\alpha}\right)^{*}}\right)$ by part (b) of Theorem 1.1. Consequently,

$$
\left\{\lambda \delta_{x}:(x, \lambda) \in X \times \mathbb{T}\right\} \subseteq \operatorname{Ext}\left(B_{\operatorname{lip}\left(X, d^{\alpha}\right)^{*}}\right) \subseteq\left\{\lambda \widetilde{\delta}_{w}:(w, \lambda) \in W \times \mathbb{T}\right\}
$$

Thus, the proof is complete.

For each $(w, \lambda) \in W \times \mathbb{T}$, we define the map $\tilde{\varphi}_{w, \lambda}: \operatorname{lip}\left(X, d^{\alpha}\right)_{r} \rightarrow \mathbb{R}$ by $\tilde{\varphi}_{w, \lambda}(f)=$ $\operatorname{Re}\left(\lambda \tilde{\delta}_{w}(f)\right)$; in fact, $\tilde{\varphi}_{w, \lambda}=\operatorname{Re}\left(\lambda\left(e_{w} O \Phi_{\alpha}\right)\right)$. Clearly, $\tilde{\varphi}_{w, \lambda} \in B_{\left(\operatorname{lip}\left(X, d^{\alpha}\right)_{r}\right)^{*}}$ for all $(w, \lambda) \in W \times \mathbb{T}$. Moreover, $\tilde{\varphi}_{x, \lambda}=\operatorname{Re}\left(\lambda \delta_{x}\right)$ for all $(x, \lambda) \in X \times \mathbb{T}$.

We now give a characterization of extreme points of the unit ball in the $\left(\operatorname{lip}\left(X, d^{\alpha}\right)_{r}\right)^{*}$ as the following.

Proposition 3.2. By above notations,

$$
\left\{\tilde{\varphi}_{x, \lambda}:(x, \lambda) \in X \times \mathbb{T}\right\} \subseteq \operatorname{Ext}\left(B_{\left(\operatorname{lip}\left(X, d^{\alpha}\right)_{r}\right)^{*}}\right) \subseteq\left\{\tilde{\varphi}_{w, \lambda}:(w, \lambda) \in W \times \mathbb{T}\right\}
$$

Further, for $(x, \lambda),(y, \mu) \in X \times \mathbb{T}$, one has $\tilde{\varphi}_{x, \lambda}=\tilde{\varphi}_{y, \mu}$ if and only if $(x, \lambda)=(y, \mu)$.

Proof. The proof is similar to that of Proposition 2.1 by replacing $\operatorname{lip}\left(X, d^{\alpha}\right), \tilde{\delta}_{x}, \tilde{\delta}_{w}, \tilde{\varphi}_{x, \lambda}, \tilde{\varphi}_{w, \lambda}$ and Theorems 1.2 and $1.3 \operatorname{by} \operatorname{Lip}\left(X, d^{\alpha}\right), \Delta_{x}, \widetilde{\Delta}_{w}, \widetilde{\psi}_{x, \lambda}, \widetilde{\psi}_{w, \lambda}$ and Theorem 3.1, respectively.

Theorem 3.3. Let $A$ be a real linear subspace of $\operatorname{lip}\left(X, \tau, d^{\alpha}\right)$ containing 1 . For $(w, \lambda) \in W \times \mathbb{T}$, let $\tilde{\theta}_{w, \lambda}=\left.\tilde{\varphi}_{w, \lambda}\right|_{A}$. Let $P=\{(x, \lambda) \in X \times \mathbb{T}: \tau(x) \neq x\} \cup\{(x, \lambda) \in X \times \mathbb{T}: \tau(x)=x, \lambda \in$ $\{-1,1\}\} \cup\{(w, \lambda) \in W \times \mathbb{T}: w \in \beta \tilde{X}\}$. Let $Q_{A}$ denote the set of all $(x, \lambda) \in X \times\{-1,1\}$ such that

(i) there is $g \in A$ with $\|g\|_{X, p_{\alpha}} \leq 1$ and $g(x)=\bar{\lambda}$,

(ii) for every $y \in X \backslash\{x, \tau(x)\}$, there is $h \in A$ with $\|h\|_{X, p_{\alpha}} \leq 1, h(x)=1$ and $|h(y)|<1$.

Then,

$$
\left\{\tilde{\theta}_{x, \lambda}:(x, \lambda) \in Q_{A}\right\} \subseteq \operatorname{Ext}\left(B_{A^{*}}\right) \subseteq\left\{\tilde{\theta}_{w, \lambda}:(w, \lambda) \in P\right\}
$$

Further, if $(x, \lambda) \in Q_{A}$ and $(y, \mu) \in P \cap(X \times \mathbb{T})$, then $\tilde{\theta}_{y, \mu}=\tilde{\theta}_{x, \lambda}$ if and only if $(y, \mu)=(x, \lambda)$ or $(y, \mu)=(\tau(x), \lambda)$.

Proof. The proof is similar to that of Theorem 2.2 by replacing $\operatorname{lip}\left(X, d^{\alpha}\right), \tilde{\theta}_{w, \lambda}, \tilde{\theta}_{x, \lambda}, \tilde{\varphi}_{x, \lambda}$, Proposition 3.2 and $\Phi_{\alpha}$ by $\operatorname{Lip}\left(X, d^{\alpha}\right), \tilde{\eta}_{w, \lambda}, \tilde{\eta}_{x, \lambda}, \widetilde{\psi}_{x, \lambda}$, Proposition 2.1 and $\Psi_{\alpha}$, respectively.

Corollary 3.4. Let $P$ be as in Theorem 3.3. For $(w, \lambda) \in P$, let $\tilde{\theta}_{w, \lambda}$ denote the restriction of $\tilde{\varphi}_{w, \lambda}$ to $\operatorname{lip}\left(X, \tau, d^{\alpha}\right)$. Then,

$$
\left\{\tilde{\theta}_{x, \lambda}:(x, \lambda) \in X \times\{-1,1\}\right\} \subseteq \operatorname{Ext}\left(B_{\operatorname{lip}(X, \tau, \alpha)^{*}}\right) \subseteq\left\{\tilde{\theta}_{w, \lambda}:(w, \lambda) \in P\right\} .
$$


Further, if $(x, \lambda) \in X \times\{-1,1\}$ and $(y, \mu) \in P \cap(X \times \mathbb{T})$, then $\theta_{y, \mu}=\tilde{\theta}_{x, \lambda}$ if and only if $(y, \mu)=(x, \lambda)$ or $(y, \mu)=(\tau(x), \lambda)$.

Proof. The proof is similar to that Corollary 2.3 by replacing $\operatorname{lip}\left(X, \tau, d^{\alpha}\right), \tilde{\theta}_{w, \lambda}, \tilde{\theta}_{x, \lambda}$ and $\operatorname{lip}\left(X, d^{\alpha}\right)$ by $\operatorname{Lip}\left(X, \tau, d^{\alpha}\right), \tilde{\eta}_{w, \lambda}, \tilde{\eta}_{x, \lambda}$ and $\operatorname{Lip}\left(X, d^{\alpha}\right)$, respectively.

\section{References}

[1] S. H. Kulkarni and B. V. Limaye, "Extreme points of the unit ball in the dual spaces of some real subspaces of C(X)," Glasnik Matematički. Serija III, vol. 29(49), no. 2, pp. 333-340, 1994.

[2] N. Dunford and J. T. Schwartz, Linear Operators. Part I: General Theory, Interscience, New York, NY, USA, 1958.

[3] S. H. Kulkarni and B. V. Limaye, "Gleason parts of real function algebras," Canadian Journal of Mathematics, vol. 33, no. 1, pp. 181-200, 1981.

[4] S. H. Kulkarni and B. V. Limaye, Real Function Algebras, vol. 168 of Monographs and Textbooks in Pure and Applied Mathematics, Marcel Dekker, New York, NY, USA, 1992.

[5] M. Grzesiak, "Extreme points of the unit ball in the dual space of some real Banach algebra of continuous complex functions," Fasciculi Mathematici, no. 16, pp. 5-10, 1986.

[6] K. de Leeuw, "Banach spaces of Lipschitz functions," Polska Akademia Nauk. Instytut Matematyczny. Studia Mathematica, vol. 21, pp. 55-66, 1961/1962.

[7] A. K. Roy, "Extreme points and linear isometries of the Banach space of Lipschitz functions," Canadian Journal of Mathematics, vol. 20, pp. 1150-1164, 1968.

[8] A. Jiménez-Vargas and M. Villegas-Vallecillos, "Into linear isometries between spaces of Lipschitz functions," Houston Journal of Mathematics, vol. 34, no. 4, pp. 1165-1184, 2008.

[9] D. Alimohammadi and A. Ebadian, "Hedberg's theorem in real Lipschitz algebras," Indian Journal of Pure and Applied Mathematics, vol. 32, no. 10, pp. 1479-1493, 2001 


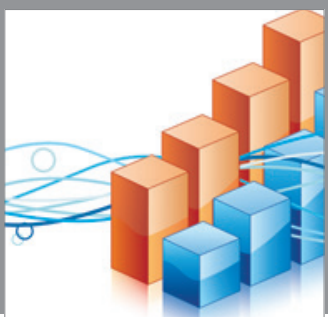

Advances in

Operations Research

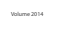

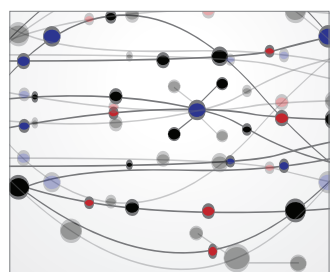

\section{The Scientific} World Journal
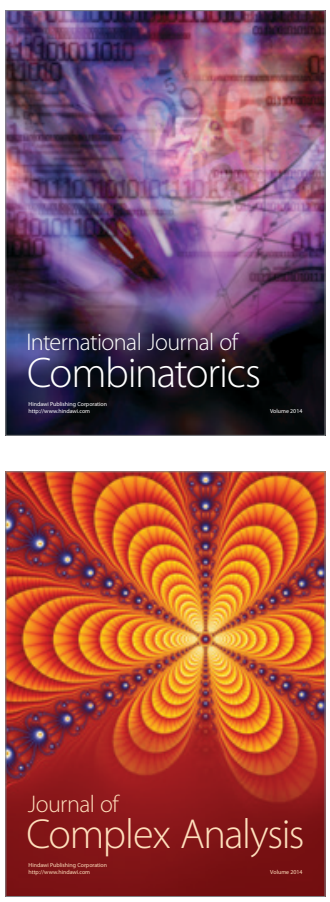

International Journal of

Mathematics and

Mathematical

Sciences
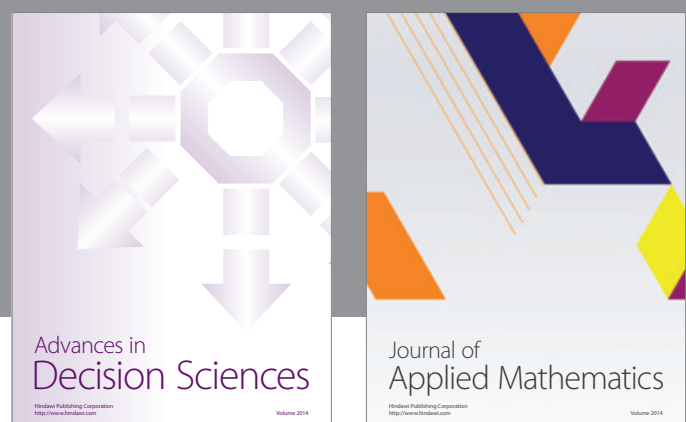

Journal of

Applied Mathematics
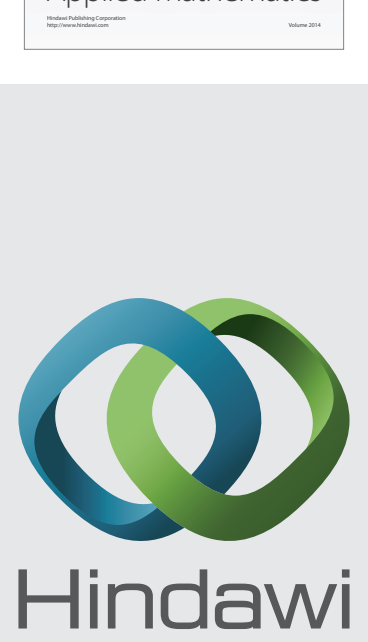

Submit your manuscripts at http://www.hindawi.com
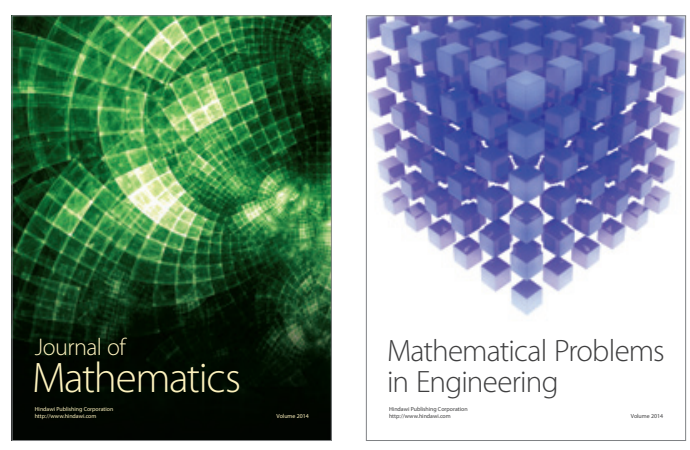

Mathematical Problems in Engineering
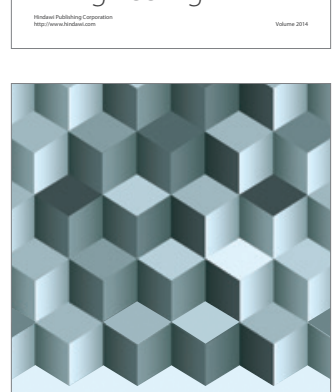

Journal of

Function Spaces
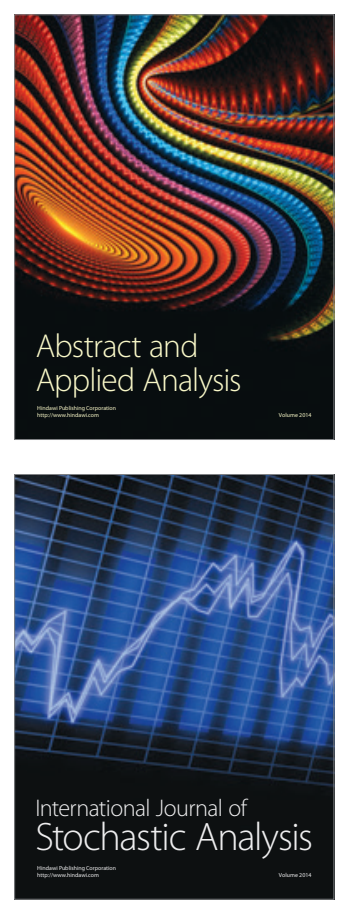

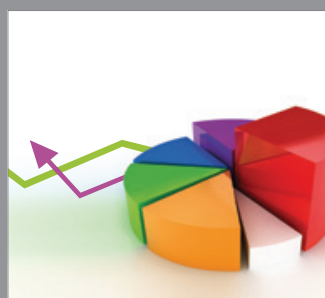

ournal of

Probability and Statistics

Promensencen
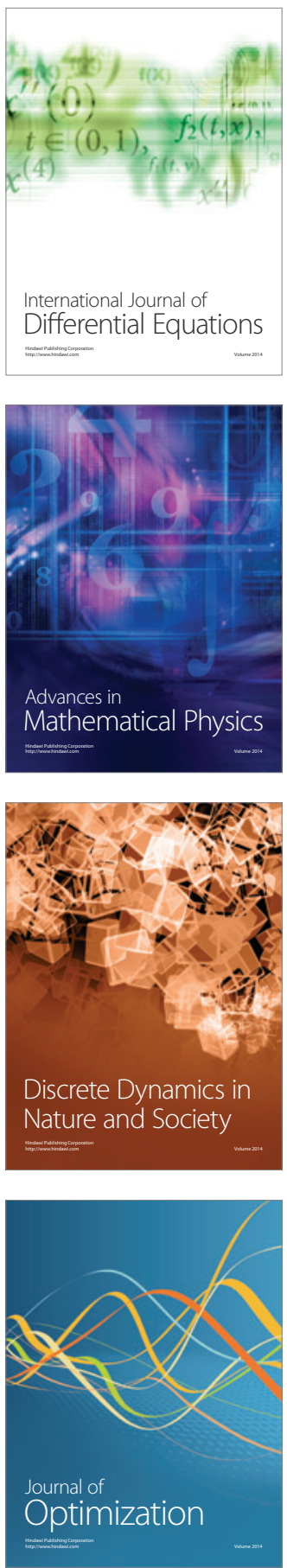